\title{
Observation of Different Isoscaling Behavior Between Emitted Fragments and Residues
}

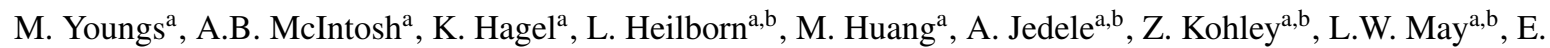 \\ McCleskey $^{a}$, A. Zarrella ${ }^{\text {a,b }}$, S.J. Yennello ${ }^{a, b}$ \\ ${ }^{a}$ Cyclotron Institute, Texas A\&M University, College Station, Texas 77843, USA \\ ${ }^{b}$ Chemistry Department, Texas AEM University, College Station, Texas 77843, USA
}

\begin{abstract}
The traditional isoscaling technique has been used to analyze all isotopically identified particles from ${ }^{70} \mathrm{Zn}+{ }^{70} \mathrm{Zn}$ and ${ }^{64} \mathrm{Zn}+{ }^{64} \mathrm{Zn}$ collisions at $35 \mathrm{MeV} / \mathrm{u}$. Two additional techniques, using an energy-averaged ratio and using the data present in the tail of energy spectra, are compared to the traditional method and show similar results. Isoscaling fit parameters $\alpha$ and $\beta$ are found both globally and for each individual series of constant $Z$ and $N$. The data are then split up between emitted fragments and projectile-like fragments. Isoscaling values for the two different types of fragments are shown to be different, emphasizing the importance of experimentally distinguishing between projectilelike fragments and emitted fragments in order to achieve accurate isoscaling parameters.
\end{abstract}

\section{Introduction}

Isoscaling is a technique which compares the ratio of the integrated yield of isotopes from one reaction system to the integrated yield from another reaction system. It is often used when the primary difference between the two systems is the isospin content [16, 19, 25]. Numerous experiments have shown that isoscaling provides reasonable fits to experimental data in a number of different types of reactions including multifragmentation [5, 10, 17, 25], evaporation [1, 2, 19], deep inelastic collisions [15, 19, 21] and fission [4, 20]. These same fits have also been shown in the results of a number of theoretical transport model calculations such as molecular dynamics [3, 12].

Traditional isoscaling fits the resulting yield ratios using the form

$$
R_{21} \equiv \frac{\left(\int \frac{d M_{2}}{d E} d E\right)}{\left(\int \frac{d M_{1}}{d E} d E\right)}=C \exp (\alpha N+\beta Z)
$$

where $C, \alpha$ and $\beta$ are fitting parameters and where $\frac{d M_{i}}{d \mathrm{E}}$ represents the spectrum as a function of kinetic energy in a chosen frame of reference from collisions of systems $i$. System 2 is typically chosen to be the more neutron rich system.

Isoscaling has been used in the past to constrain the nuclear symmetry energy and has been improved upon by using quasi-projectile reconstruction to choose very narrow ranges of sources [11, 22]. This method unfortunately adds significant statistical constraints due to the strict requirements needed to reconstruct a quasi-projectile. With the exception of a few select cases such as Refs. [14, 17, 22], experimental results have typically been limited to small ranges of isotopes within a given element based primarily on statistics. Some conditions and limits for isoscaling have been studied in Ref [18], where the authors found that isoscaling does not necessarily provide details about the reaction mechanism. In addition, they concluded that the temperatures of the reaction systems must be similar or a correction for the difference in temperature must be made, if a thermodynamic model is being applied to interpret the data. The present work investigates constraints in isoscaling when larger and more exotic isotopes are included. In this case, instead of using isoscaling to obtain fit values from a specific type of reaction, this work seeks to investigate the magnitude of the effect of including heavy source remnants with emitted fragments in the isoscaling analysis. In addition, we investigate the differences in behavior between heavy and light particles in regards to isoscaling. 


\section{Experiment}

The systems selected for this analysis were symmetric collisions of ${ }^{70} \mathrm{Zn}+{ }^{70} \mathrm{Zn}$ and ${ }^{64} \mathrm{Zn}+{ }^{64} \mathrm{Zn}$ at $E / A=35 \mathrm{MeV}$. These reactions were chosen in order to measure products from sources with similar temperatures and charge yet significantly different isospin. Specifics about the experiment as well as its calibration and particle identification are described in depth in Refs. [7-99]. Charged particles were measured using the NIMROD array [13, 23, 24]. NIMROD is a $4 \pi$ charged particle and neutron detector that is typically capable of isotopic resolution up to $Z=17$. Certain telescopes attained isotopic resolution up through $Z=20$. The device is also capable of elemental resolution up to the charge of the beam $(Z=30)$. In addition, free neutron multiplicity was measured in this device and was used in the determination of an impact parameter surrogate.

\section{Data Selection}

In this analysis, only the ratios of spectra of a given composite fragment from one reaction system to the other are considered. The differential efficiency between the two reaction systems in question was minimized by considering only the detectors which behave similarly between the two systems. Assuming that this accounts for all but slight differential efficiencies, the particle ratios as a function of energy will be accurate without the need for full efficiency considerations which can be an immensely time consuming process for little gain in precision for the $R_{21}$ observable.

To compare similar reaction mechanisms, an impact parameter surrogate was used to select on the violence of a collision. The violence of the collision is determined using both the free neutron and charged particle multiplicities. The statistics are evenly divided into five bins where the events with the largest amount of free neutrons and charged particles are assumed to be the most violent and thus most central collisions as described in Ref. [7].

In addition to selecting events based on the charged particle and neutron multiplicities, events are also chosen based on the total charge detected. Due to the $4 \pi$ nature of the NIMROD array and the ability to elementally identify particles up through the charge of the beam, it is possible to require that most of the projectile charge is detected. For this analysis, events are required to have measured at least $70 \%$ of the charge of the beam which corresponds to a total charge measured of $Z=21$. NIMROD is not designed to measure target-like fragments. It is effective in analysis of NIMROD data to select on properties of the fast moving nuclei such as the fragments of the projectile for event characterization. Particles that are elementally identified are included in the total $Z$ requirement whether or not they are isotopically identified. From these events, only isotopically identified particles are included in the spectra and ratios.

The energy dependent ratios are investigated as a function of the violence bin. Figure 1 shows these ratios which are represented as the $R_{21}$ value as a function of center of mass kinetic energy of the fragment cut on the violence bin for ${ }^{12} \mathrm{C},{ }^{17} \mathrm{O}$ and ${ }^{23} \mathrm{Ne}$. For each isotope, the results from the most violent cut and thus most central collisions are represented as Cut 0 (black circles), while the least violent and most peripheral collisions are represented as Cut 4 (green stars). For each of the isotopes, the $R_{21}$ value is nearly identical as a function of energy for each of the violence cuts.

This result differs from the Le Fevre [10] data which showed a dependence of the isoscaling parameter $\alpha$ using Equation 1 for different impact parameter selections. The most likely explanation for this discrepancy is described in Ref. [7]. In that work, molecular dynamics simulations were run over a large impact parameter range. The results of those simulations were then filtered to mimic an experimentally determined violence cut. The pertinent result found a significant overlap in impact parameter between each of the violence cuts. This overlap indicates significant event mixing between each of the violence bins and may explain the lack of a difference between the $R_{21}(E)$ for each violence cut. The Le Fevre experiment also used collisions at significantly higher energy which allowed a more sensitive calculation of the impact parameter and likely less event mixing between their cuts. Due to the fact that the $R_{21}$ values for all these violence cuts are nearly identical, and that the demonstration of the effect of mixing heavy and emitted particles is the main scope of this work, the spectra from all violence cuts are summed together in order to achieve a larger statistical sample for all isotopes, especially in the larger and more exotic species.

\section{Experimental Results}

Upon investigation of the energy dependent ratios for different particles we note a variety of effects. Figure 2 shows the ratios for a wide variety of isotopes from boron through magnesium. Isotopes with $Z \leq 4$ were omitted due 


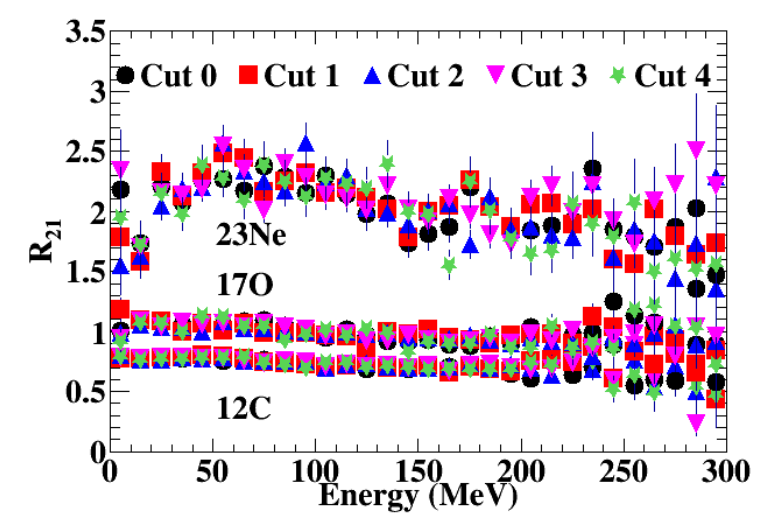

Figure 1: (Color online) The energy dependent ratios for ${ }^{12} \mathrm{C}$ (bottom), ${ }^{17} \mathrm{O}$ (middle) and ${ }^{23} \mathrm{Ne}$ (top). Cut 0 represents the most violent collisions and imply the most central reactions while Cut 4 represents the least violent, most peripheral reactions. Cuts are chosen to give nearly equal number of events in each of the five bins.

to the effects of secondary decay. Each panel in this figure represents a set of isotopes that have the same value of $N-Z$. In this figure there are several important trends. As one moves from panel to panel, with an increasing value of $N-Z$, the values of the ratios increase. In addition, the ratios do not have a significant dependence on the energy.

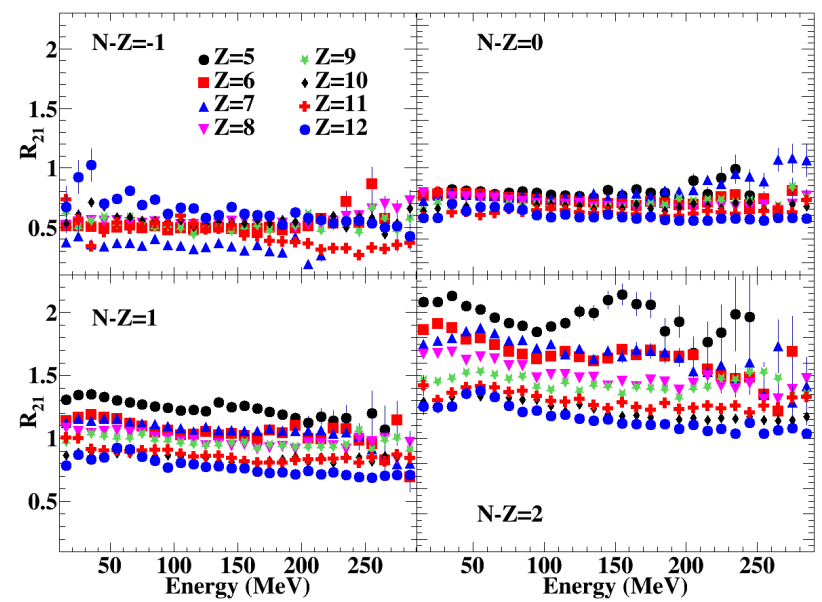

Figure 2: (Color online) The ratios of the kinetic energy spectra as a function of energy. Different panels display isotopes with particular values of $N-Z$. The legend in the upper left panel applies to all four panels. Measured isotopes for boron through magnesium are shown.

The final trend can be found in all the panels but becomes most obvious in the lower right, $N-Z=2$, panel. Given a constant value of $N-Z$, the ratio decreases as the $Z$ of the isotope increases. This provides an immediate and interesting insight into the relationship between the $\alpha$ and $\beta$ fit parameters. As the neutron value increases within a set of constant $Z$, the yield ratio increases, requiring a positive $\alpha$ value as expected. As the proton value increases within a set of constant $N$, the yield ratio decreases requiring a negative $\beta$ value, again as expected. Lastly, the fact that the yield ratio decreases with increasing mass requires the magnitude of $\beta$ to be larger than that of $\alpha$, which is consistent with the results found in Ref. [27].

While the energy dependence of the ratios is relatively small, most of these isotopes do indeed have a slope between $0 \mathrm{MeV}$ and $300 \mathrm{MeV}$. One possible explanation for this would be a difference in the average temperatures between the two sets of reactions. If this were the case it would be expected that all the ratios would have a similar positive or negative slope depending on which source had a higher average temperature.

Figure 3 provides a comparison of the four most abundantly measured isotopes of carbon, oxygen and neon. Each 


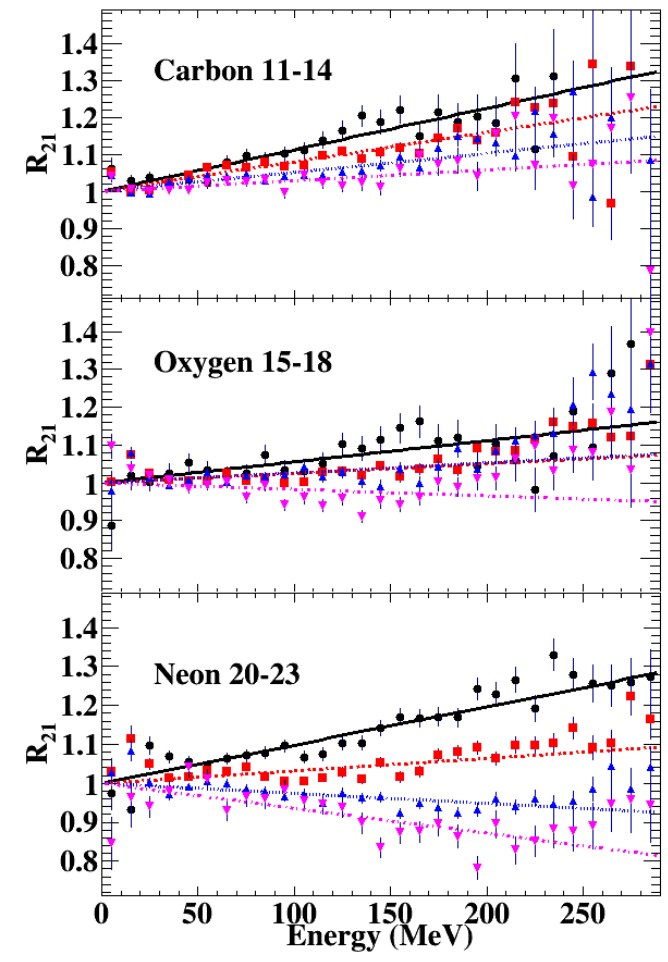

Figure 3: (Color online) The energy dependent ratios and a fit line for several isotopes of carbon (top), oxygen (middle) and neon (bottom). In each case the mass of the isotopes increases in the order of black circles, red squares, blue triangles and pink inverted triangles. As the mass increases, the slope decreases.

\begin{tabular}{|c|c||c|c||c|c|}
\hline Isotope & Slope & Isotope & Slope & Isotope & Slope \\
\hline $11 \mathrm{C}$ & 1.10 & $15 \mathrm{O}$ & 0.612 & $20 \mathrm{Ne}$ & 1.03 \\
\hline $12 \mathrm{C}$ & 0.874 & $16 \mathrm{O}$ & 0.369 & $21 \mathrm{Ne}$ & 0.399 \\
\hline $13 \mathrm{C}$ & 0.581 & $17 \mathrm{O}$ & 0.304 & $22 \mathrm{Ne}$ & -0.141 \\
\hline $14 \mathrm{C}$ & 0.344 & $18 \mathrm{O}$ & -0.116 & $23 \mathrm{Ne}$ & -0.440 \\
\hline
\end{tabular}

Table 1: The slopes of the fit lines for the isotopes from Figure 3 All the slope values have been multiplied by a factor of 1000

isotope was fit to a line and subsequently vertically shifted to clearly compare the slopes. The shift is such that the fit to the ratio is defined to be 1.0 at $0 \mathrm{MeV}$. The lightest mass of each element is shown with black circles with increasing mass represented, in order, by red squares, blue triangles and pink inverted triangles. Table 1 shows the slopes of the fit lines for each isotope. The values provided have been multiplied by a factor of 1000 . Unexpectedly, as the neutron number increases the slope of the ratio decreases within a given element. This is very different than one might expect if the systems simply had different average temperatures.

This result immediately brings into question the accuracy of finding the isoscaling parameters using the full integrated yields. These slopes suggest that the tails of these spectra may have some increased sensitivity to perturbative effects between the two reactions, such as slight differences in Coulomb, pairing and secondary decay effects. If this is the case, the average of the energy dependent ratio or using the integrals of only the tails of the spectra may also show an increased sensitivity to these effects.

The standard construction, $R_{21, s t d}$ is defined to be the ratio of the integral of the yields over all energies as shown in Equation 1. We define a similar treatment, $R_{21, \text { tail }}$ to be the ratio of the integral of the yields in the tails of the spectra. The energy range of the tail was chosen to be the range in which the spectrum falls below $30 \%$ of the yield at the peak height. The final method weights each energy bin equally and is the average value of the ratio over all energies, $R_{21, \text { ave }} \equiv\left\langle R_{21}(E)\right\rangle$ where $R_{21}(E)$ is now an energy-dependent ratio. Figure 4 shows a metric of the difference between 


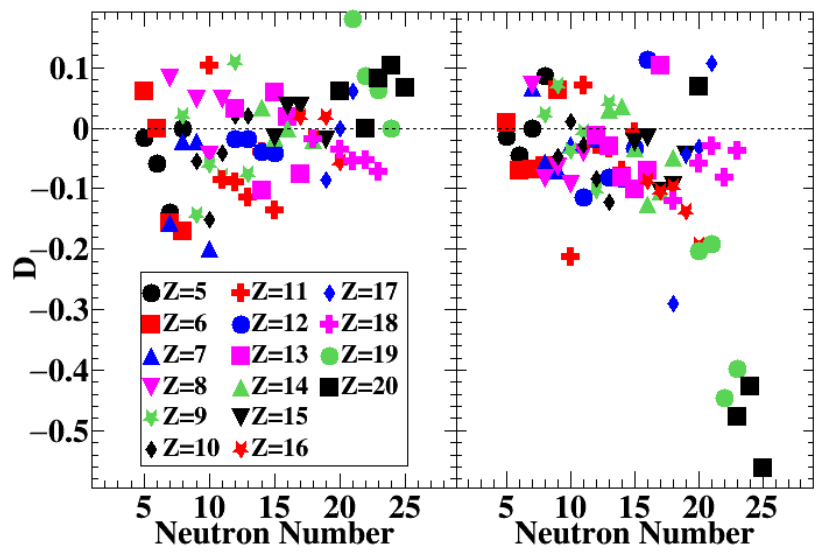

Figure 4: (Color online) The difference between the $R_{21, \text { ave }}$ (left) and $R_{21, \text { tail }}$ (right) and $R_{21, \text { std }}$ for each of the measured isotopes.

the $R_{21, \text { ave }}$ (left) and $R_{21, \text { tail }}$ (right) with $R_{21, \text { std }}$ for each of the measured isotopes. The vertical axis is calculated using

$$
D=\frac{R_{21, i}-R_{21, s t d}}{R_{21, s t d}}
$$

where $i$ represents the two alternate methods of calculating the ratio. Both of these constructions have the potential to be more sensitive than the standard method. The $R_{21, a v e}$ value weights each energy bin equally and so may provide more sensitivity to the slopes shown in Figure 3 . The $R_{21 \text {,tail }}$ construction represents an almost negligible amount of statistics compared to the full integral and as such this method should be able to better identify sensitivity to any effects that exist in that energy region. In both cases the difference between these new methods and the standard method are less than $20 \%$ for all isotopes with the exception of the tail construction for the heaviest few isotopes. While Figure 3 suggests an effect in the tail of the energy distribution, Figure 4 does not corroborate this. It is possible that this effect does indeed exist but these two methods are not sufficient or sensitive enough to see it with the current level of statistics. Since there is not a consistent, noticeable difference between the methods, all subsequent analysis and figures will use the $R_{21, \text { std }}$ values.

The data presented up to this point has included all particles that were isotopically identified in the NIMROD array. The original model of isoscaling was intended to be used for particles that were emitted from an excited source. In this respect it is illogical to consider emitted fragments with those that are the evaporation residues, i.e. the projectile-like fragments (PLF). The PLF was determined in each event as the largest particle detected with a mass of $A \geq 9$. When there were no particles of sufficient mass it was assumed that the PLF was not detected due to imperfect coverage since analyzed events required $\sum Z \geq 21$. It is possible that the heaviest particle in an event was measured in a detector that could only elementally identify the particle instead of isotopically identify it. These heavy particles were marked as the PLF but were not considered in the calculation of $R_{21}$ values. It should be noted that this definition of the PLF is rather simplistic. In an effort to maximize the statistics and to demonstrate the effects of mixing the residues and the emitted particles, no cuts have been made to determine the PLF other than mass. This definition of the PLF should be understood as the residue of an emitting source.

The $R_{21}$ values for all (left), PLF (center) and emitted (right) particles are presented in Figure 5 . The top row and bottom row show the same data with two different fits. The top row shows the global isoscaling fit while the bottom row shows the individual fits for each element. In order to compare to previously published isoscaling data, both the global and individual fits use the three most abundant isotopes in order to find the isoscaling $\alpha$ and $\beta$ values from Equation 1. In the figure, the isotopes used for the fit are provided in solid symbols while the isotopes that were not considered are provided with open symbols.

This figure shows several distinct trends. The primary trend is that the ratio for emitted fragments can be significantly different than the ratio for PLFs with the emitted fragments having $R_{21}$ values sometimes as much as double the value for the PLF of the same isotope. In addition, the PLF fragments show a significant deviation from the simple linear behavior on this semi-log plot. This is most noticeable in the masses larger than those used for the fits. In 


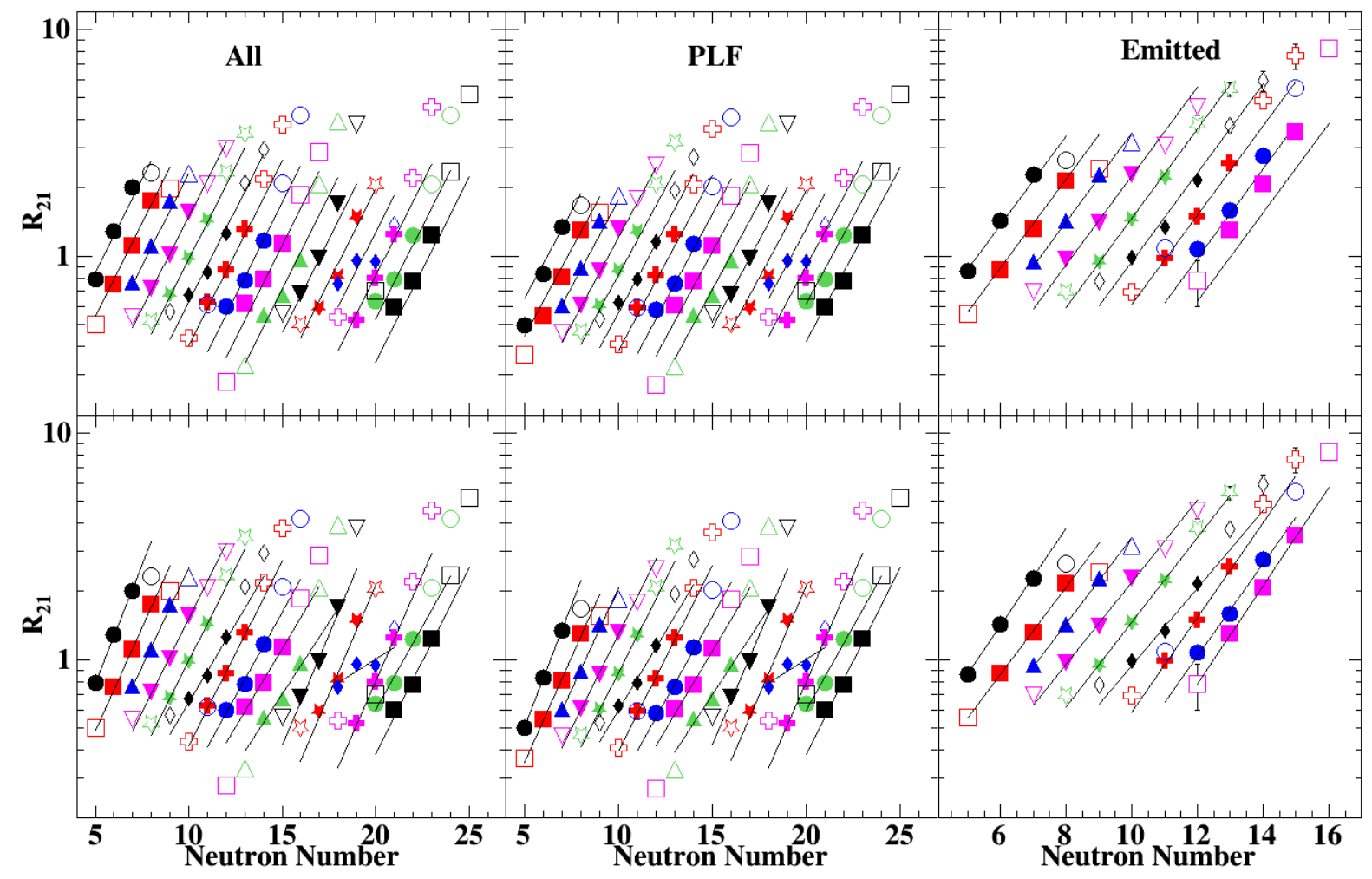

Figure 5: (Color online) The $R_{21, s t d}$ values using all particles (left), heavy PLFs (middle) and light emitted particles (right). The isotopes used range from boron (solid black circle) to calcium (solid black squares). Elements proceed in order from left to right with each color and symbol combination representing a specific element. The legend is the same as Figure 4 The top row also provides the lines that correspond to fit results of a single global fit. The bottom row shows the same data values with individual fits for each element. It is important to note the change in range for the $x$-axis in the rightmost column.

fact, this behavior is similar to the parabolic behavior seen in Ref. [18]. However, attention should be directed to the more massive isotopes. When these heavy particles are measured they are statistically more likely to be the heaviest fragment in an event and thus defined to be the PLF. In the rare cases when they are determined to be an emitted fragment, the particles show a less extreme curvature as a function of neutron number within a given element.

Chlorine $(Z=17)$ is the only element that shows a drastically different slope, and thus $\alpha$ value, from the others. Wuenschel et al. [22] saw similar effects in chlorine from a different set of reaction systems also measured on the NIMROD array. Using a quasi-projectile reconstruction method that includes free neutrons in the reconstruction, they found isoscaling parameters for chlorine that are similar to the rest of the elements. At present, the reason for this behavior of chlorine is unknown. Since this seems to be an effect specific to chlorine, those isotopes were eliminated from consideration for the fits for individual $\beta$ values as well as the global $\alpha$ and $\beta$ fits.

Figure 6 shows the results of individual fits for each $Z$ and $N$ for both emitted fragments and PLFs. The black short dashed line, red dot-and-dash line and blue long dash line represent the global $\alpha$ and $\beta$ values for the emitted, PLF and all fragments, respectively. The solid line at zero indicates the separation in $\alpha$ and $\beta$. In addition to the $R_{21}$ values being larger for emitted fragments than PLFs, the $\alpha$ values are also consistently found to be larger.

Figure 6 shows some of the benefits and potential downfalls of the traditional isoscaling technique. In the case of the parameter $\alpha$, the PLF global fit has a similar value to the global fit using all particles. Those two fits provide $\alpha$ values significantly smaller than the value found for emitted particles. In fact the emitted particle value is nearly $30 \%$ larger than the PLF value. While comparing the results for $\beta$, we find that the emitted and all particle values are nearly identical while being rather different than the PLF value. This indicates that isoscaling parameters found using all measured particles could be inaccurate. 


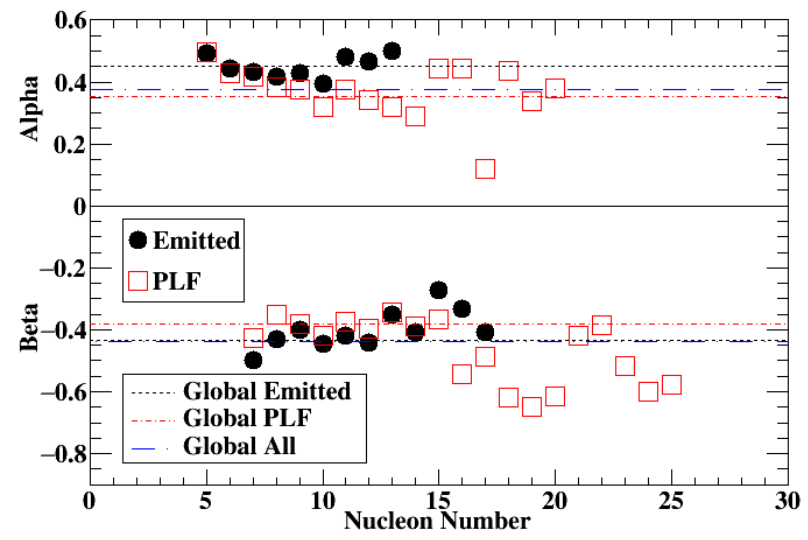

Figure 6: (Color online) The isoscaling parameters $\alpha$ and $\beta$ for both emitted particles and PLFs. The global fit values for the emitted, PLF and all particles are also included as well as a line at zero to separate the $\alpha$ and $\beta$ values.

In addition to this, the previous derivation of the relative magnitudes between $\alpha$ and $\beta$ was based on the observations in Figure 2 that used all particles. In the case of the emitted particles, however, the global fit finds that the magnitudes of $\alpha$ and $\beta$ are much closer to each other. With these new results in mind, one would expect that the separation found in Figure 2 would be significantly smaller if only emitted particles were used. Figure 7 shows exactly this result. In fact, the right-most column of Figure 5 shows this as well. The $R_{21}$ ratios for a given $N-Z$ are significantly closer to each other than compared to the PLF which shows a decreasing trend as $Z$ increases. This is an important result for future studies of isoscaling. In this experiment, the global $\alpha$ value for emitted particles is nearly $30 \%$ larger than that found for all detected particles. This result emphasizes the importance of being able to determine whether a particle was emitted from a source or whether it was the PLF in order to accurately find the isoscaling parameters.

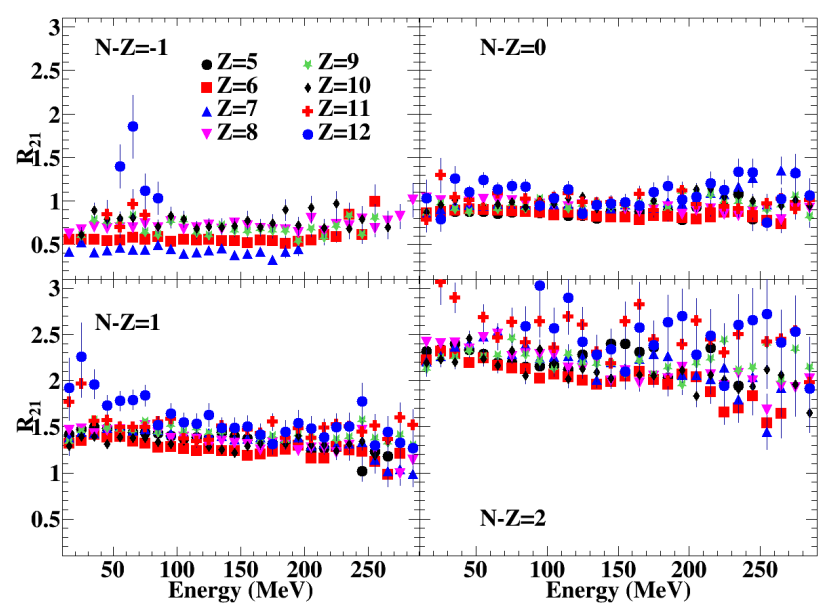

Figure 7: (Color online) The ratios of kinetic energy spectra as a function of energy. Different panels display isotopes with different values of $N-Z$. The legend in the upper left panel applies to all four panels. $R_{21}$ values shown here are constructed from only emitted particles instead of all measured particles as in Figure 2

\section{Summary and Discussion}

In summary, isoscaling using integrated yields was measured for all isotopically identified particles from a wide variety of sources and classes of events from reactions of ${ }^{70} \mathrm{Zn}+{ }^{70} \mathrm{Zn}$ and ${ }^{64} \mathrm{Zn}+{ }^{64} \mathrm{Zn}$ at $E / A=35 \mathrm{MeV}$. The results suggest that there are a number of effects that should be considered in order to achieve a more physically accurate 
set of isoscaling fit parameters. Two main effects are the kinetic energy dependence of the $R_{21}$ value as a function of neutron number and the difference in $R_{21}, \alpha$ and $\beta$ determined from PLFs and emitted fragments.

The lack of a significant energy dependence in the yield ratio may be explained through a basic thermodynamic model. The thermodynamic model of heavy ion collisions as described by das Gupta and Mekjian [6] predicts the shape of the energy distribution of composite nuclei based on that of protons. The differential multiplicity of a fragment is described by

$$
\frac{d^{3} M_{A}}{d^{3} p_{A}}=\exp \left(\frac{\mu_{A}}{T}\right) g_{A} \frac{V}{h^{3}} \exp \left(-\frac{\sqrt{m_{A}^{2} c^{4}+p^{2} c^{2}}}{T}\right)
$$

where the chemical potential $\mu_{A}=N \mu_{n}+Z \mu_{p}$ for a composite particle of mass $A$ that has $N$ neutrons and $Z$ protons. Here, $g_{A}$ is the spin degeneracy factor of the composite, $\mu_{n, p}$ are the chemical potentials from the emitting source for neutrons and protons respectively, $V$ is the volume and $T$ is the temperature. Using Equation 3 , we can take the ratio of the energy spectrum for a chosen isotope from one reaction system to the energy spectrum for the same isotope from a different reaction system. Typically these systems are selected such that the source will not have a noticeable difference in temperature or size. In addition, the systems are chosen so that the source charge will not be significantly different in order to minimize Coulomb effects. If these conditions are true, as in this experiment, then taking the ratio of spectra for a specific isotope from the two systems will cause most of the terms in Equation 3 to cancel out leaving

$$
R_{21} \equiv \frac{\left(\frac{d M_{2}}{d E}\right)}{\left(\frac{d M_{1}}{d E}\right)}=C^{\prime} \exp (\Delta \mu / T)
$$

where the difference in the chemical potential between the two systems is defined as $\Delta \mu=N \Delta \mu_{n}+Z \Delta \mu_{p}$. If the systems are at the same temperature, the value of the $R_{21}$ ratio depends only on the difference between these two sources in the chemical potentials for both neutrons and protons. This equation can also be related to Equation 1 where $\alpha$ is proportional to $\Delta \mu_{n} / T$ and $\beta$ is proportional to $\Delta \mu_{p} / T$.

A simple description of a spherical nucleus will show that the change in chemical potential between two nuclei where the only difference between them is the neutron number (and thus the volume) is equal in magnitude and opposite in sign for protons and neutrons. This would imply the expectation of the $\alpha$ and $\beta$ values being equal and opposite. The global fits for emitted particles demonstrates this prediction reasonably well.

This is, of course, a relatively primitive model. There are small effects that can change the chemical potential for protons and neutrons differently in different systems such as Coulomb, pairing and structure effects as well as density fluctuations. In each of these cases, however, the difference between the two systems should be rather small and thus these effects should not cause a significant difference. This data considering only emitted fragments shows evidence for this. While it is possible, it is also unlikely that any of these would affect the energy dependence of these ratios.

Secondary decay is more likely to cause an energy dependence in these ratios. The model used in Equation 3 assumes that the spectra are comprised of composite particles that are not excited. Due to this simplicity, there is no mechanism for particles to decay after emission, which is not the case in a true experiment in which we measure end of decay chain particles.

As previously mentioned, the isotopes smaller than boron were omitted due to these secondary decay effects. There have been some previous studies on the effect of secondary decay as they pertain to isoscaling such as in Ref [26]. In these results, the authors suggest that particles with $Z \leq 4$ behave differently due to the effect of secondary decay. In their work, they find that the isoscaling parameters $\alpha$ and $\beta$ do indeed change when comparing their simulation results which have decayed a single time to a fully cooled set of decays for particles with $Z \geq 5$. The parameters for individual fits did, however, change by a similar amount for each element and isotone suggesting the continued validity of the isoscaling method as a means of finding global trends as it was intended, even with the effects of secondary decay.

In conclusion, the difference in $R_{21}$ and the isoscaling parameters $\alpha$ and $\beta$ between the PLFs and emitted fragments is an important result for future studies of isoscaling, in particular the choice of detector and collision system combination. With the choice of $\mathrm{Zn}+\mathrm{Zn}$, the dominant amount of events in which oxygen through aluminum were measured were events where these fragments were the largest particle measured and thus defined as the PLF. Without separating the PLF from the emitted fragments, isoscaling that included these isotopes would be driven by the PLF, which provides an inaccurate result with respect to the agreement between the two fitting parameters. 
In significantly larger systems, such as $\mathrm{Sn}+\mathrm{Sn}$, it is possible that this selection of isotopes would better represent emitted fragments instead of PLFs. In this case it would likely be enough to measure up through these fragments instead of requiring full elemental detection up through the beam. However, these results do suggest the importance of distinguishing between PLFs and emitted particles for accurate isoscaling fit parameters in the $\mathrm{Zn}+\mathrm{Zn}$ systems and for systems of similar size. In fact, to obtain the most precise isoscaling values, a reconstruction of the emitting source is likely required for each event. If an experiment is not seeking to be quite so precise and looks to investigate some more general trends, then at the very least the PLF-type fragments must be omitted from the isoscaling fits.

\section{Acknowledgements}

This work was supported by the Robert A. Welch Foundation (Grant A-1266) and the United States Department of Energy (Grant DE-FG02-93ER40773).

[1] A. S. Botvina, O. V. Lozhkin, and W. Trautmann. Isoscaling in light-ion induced reactions and its statistical interpretation. Phys. Rev. C, 65:044610, Mar 2002.

[2] J. Brzychczyk, D. S. Bracken, K. Kwiatkowski, K. B. Morley, E. Renshaw, and V. E. Viola. Isotopic yields of intermediate-mass fragments emitted in $E / A=50 \mathrm{mev}{ }^{4} \mathrm{He}+{ }^{116,124}$ sn reactions. Phys. Rev. C, 47:1553-1562, Apr 1993.

[3] C. O. Dorso, C. R. Escudero, M. Ison, and J. A. López. Dynamical aspects of isoscaling. Phys. Rev. C, 73:044601, Apr 2006.

[4] W. A. Friedman. Isotopic yields and isoscaling in fission. Phys. Rev. C, 69:031601, Mar 2004

[5] E. Geraci, M. Bruno, M. D’Agostino, E. De Filippo, A. Pagano, G. Vannini, M. Alderighi, A. Anzalone, L. Auditore, V. Baran, R. Barn, M. Bartolucci, I. Berceanu, J. Blicharska, A. Bonasera, B. Borderie, R. Bougault, J. Brzychczyk, G. Cardella, S. Cavallaro, A. Chbihi, J. Cibor, M. Colonna, D. De Pasquale, M. Di Toro, F. Giustolisi, A. Grzeszczuk, P. Guazzoni, D. Guinet, M. Iacono-Manno, A. Italiano, S. Kowalski, E. La Guidara, G. Lanzalone, G. Lanzan, N. Le Neindre, S. Li, S. Lo Nigro, C. Maiolino, Z. Majka, G. Manfredi, T. Paduszynski, M. Papa, M. Petrovici, E. Piasecki, S. Pirrone, G. Politi, A. Pop, F. Porto, M.F. Rivet, E. Rosato, S. Russo, P. Russotto, G. Sechi, V. Simion, M.L. Sperduto, J.C. Steckmeyer, A. Trifir, M. Trimarchi, M. Vigilante, J.P. Wieleczko, J. Wilczynski, H. Wu, Z. Xiao, L. Zetta, and W. Zipper. Isoscaling in central 124sn+64ni, 112sn+58ni collisions at 35 a mev. Nuclear Physics A, 732:173 - 201, 2004.

[6] S. Das Gupta and A.Z. Mekjian. The thermodynamic model for relativistic heavy ion collisions. Physics Reports, $72(3): 131-183,1981$.

[7] Z. Kohley. PhD thesis, Texas A\&M University, 2010.

[8] Z. Kohley, A. Bonasera, S. Galanopoulos, K. Hagel, L. W. May, A. B. McIntosh, B. C. Stein, G. A. Souliotis, R. Tripathi, S. Wuenschel, and S. J. Yennello. Correlations with projectile-like fragments and emission order of light charged particles. Phys. Rev. C, 86:044605, Oct 2012.

[9] Z. Kohley, L. W. May, S. Wuenschel, M. Colonna, M. Di Toro, M. Zielinska-Pfabe, K. Hagel, R. Tripathi, A. Bonasera, G. A. Souliotis, D. V. Shetty, S. Galanopoulos, M. Mehlman, W. B. Smith, S. N. Soisson, B. C. Stein, and S. J. Yennello. Transverse collective flow and midrapidity emission of isotopically identified light charged particles. Phys. Rev. C, 83:044601, Apr 2011.

[10] A. Le Fèvre, G. Auger, M. L. Begemann-Blaich, N. Bellaize, R. Bittiger, F. Bocage, B. Borderie, R. Bougault, B. Bouriquet, J. L. Charvet, A. Chbihi, R. Dayras, D. Durand, J. D. Frankland, E. Galichet, D. Gourio, D. Guinet, S. Hudan, G. Immé, P. Lautesse, F. Lavaud, R. Legrain, O. Lopez, J. Łukasik, U. Lynen, W. F. J. Müller, L. Nalpas, H. Orth, E. Plagnol, G. Raciti, E. Rosato, A. Saija, C. Schwarz, W. Seidel, C. Sfienti, B. Tamain, W. Trautmann, A. Trzciński, K. Turzó, E. Vient, M. Vigilante, C. Volant, B. Zwiegliński, and A. S. Botvina. Isotopic scaling and the symmetry energy in spectator fragmentation. Phys. Rev. Lett., 94:162701, Apr 2005.

[11] L. May. PhD thesis, Texas A\&M University, 2015.

[12] Akira Ono, P. Danielewicz, W. A. Friedman, W. G. Lynch, and M. B. Tsang. Isospin fractionation and isoscaling in dynamical simulations of nuclear collisions. Phys. Rev. C, 68:051601, Nov 2003.

[13] R.P. Schmitt, L. Cooke, G. Derrig, D. Fabris, B. Hurst, J.B. Natowitz, G. Nebbia, D. O'Kelly, B.K. Srivastava, W. Turmel, D. Utley, H. Utsunomiya, and R. Wada. Nuclear Instruments and Methods in Physics Research Section A: Accelerators, Spectrometers, Detectors and Associated Equipment, 354(23):487 - 495, 1995.

[14] G. A. Souliotis, D. V. Shetty, A. Keksis, E. Bell, M. Jandel, M. Veselsky, and S. J. Yennello. Heavy-residue isoscaling as a probe of the symmetry energy of hot fragments. Phys. Rev. C, 73:024606, Feb 2006.

[15] G. A. Souliotis, D. V. Shetty, M. Veselsky, G. Chubarian, L. Trache, A. Keksis, E. Martin, and S. J. Yennello. Isotopic scaling of heavy projectile residues from the collisions of $25 \mathrm{MeV} /$ nucleon ${ }^{86} \mathrm{Kr}$ with ${ }^{124} \mathrm{Sn},{ }^{112} \mathrm{Sn}$ and ${ }^{64} \mathrm{Ni},{ }^{58} \mathrm{Ni}$. Phys. Rev. C, 68:024605, Aug 2003.

[16] W. P. Tan, B.-A. Li, R. Donangelo, C. K. Gelbke, M.-J. van Goethem, X. D. Liu, W. G. Lynch, S. Souza, M. B. Tsang, G. Verde, A. Wagner, and H. S. Xu. Fragment isotope distributions and the isospin dependent equation of state. Phys. Rev. C, 64:051901, Sep 2001.

[17] D. Henzlova et al. arXiv:nucl-ex/0507003, 2010.

[18] M. B. Tsang, W. A. Friedman, C. K. Gelbke, W. G. Lynch, G. Verde, and H. S. Xu. Conditions for isoscaling in nuclear reactions. Phys. Rev. $C, 64: 041603$, Sep 2001 .

[19] M. B. Tsang, W. A. Friedman, C. K. Gelbke, W. G. Lynch, G. Verde, and H. S. Xu. Isotopic scaling in nuclear reactions. Phys. Rev. Lett., 86:5023-5026, May 2001.

[20] M. Veselsky, G. A. Souliotis, and M. Jandel. Isoscaling studies of fission: A sensitive probe into the dynamics of scission. Phys. Rev. C, 69:044607, Apr 2004

[21] V.V. Volkov. Deep inelastic transfer reactions the new type of reactions between complex nuclei. Physics Reports, 44(2):93 - 157, 1978.

[22] S. Wuenschel, R. Dienhoffer, G. A. Souliotis, S. Galanopoulos, Z. Kohley, K. Hagel, D. V. Shetty, K. Huseman, L. W. May, S. N. Soisson, B. C. Stein, A. L. Caraley, and S. J. Yennello. Isoscaling of fragments with $z=1-17$ from reconstructed quasiprojectiles. Phys. Rev. C, 79:061602, Jun 2009 
[23] S. Wuenschel, K. Hagel, R. Wada, J.B. Natowitz, S.J. Yennello, Z. Kohley, C. Bottosso, L.W. May, W.B. Smith, D.V. Shetty, B.C. Stein, S.N. Soisson, and G. Prete. Nimrodisis, a versatile tool for studying the isotopic degree of freedom in heavy ion collisions. Nuclear Instruments and Methods in Physics Research Section A: Accelerators, Spectrometers, Detectors and Associated Equipment, 604(3):578 - 583, 2009.

[24] Sara Wuenschel. PhD thesis, Texas A\&M University, 2009.

[25] H. S. Xu, M. B. Tsang, T. X. Liu, X. D. Liu, W. G. Lynch, W. P. Tan, A. Vander Molen, G. Verde, A. Wagner, H. F. Xi, C. K. Gelbke, L. Beaulieu, B. Davin, Y. Larochelle, T. Lefort, R. T. de Souza, R. Yanez, V. E. Viola, R. J. Charity, and L. G. Sobotka. Isospin fractionation in nuclear multifragmentation. Phys. Rev. Lett., 85:716-719, Jul 2000.

[26] P. Zhou, W. D. Tian, Y. G. Ma, X. Z. Cai, D. Q. Fang, and H. W. Wang. Influence of statistical sequential decay on isoscaling and symmetry energy coefficient in a gemini simulation. Phys. Rev. C, 84:037605, Sep 2011.

[27] P. Marini, A. Bonasera, A. McIntosh, R. Tripathi, S. Galanopoulos, K. Hagel, L. Heilborn, Z. Kohley, L. W. May, M. Mehlman, S. N. Soisson, G. A. Souliotis, D. V. Shetty, W. B. Smith, B. C. Stein, S. Wuenschel, and S. J. Yennello. Constraining the symmetry term in the nuclear equation of state at subsaturation densities and finite temperatures. Phys. Rev. C, 85:034617, Mar 2012. 